\section{SILICON PHOTONICS}

\section{Wire-bonding assembly \\ Optica 5, 876-883 (2018)}

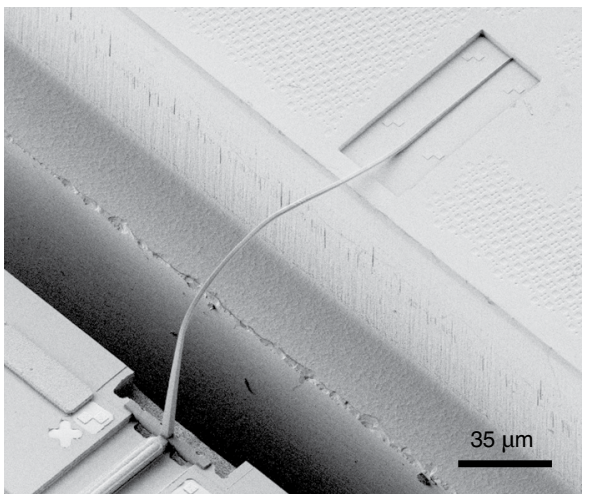

Credit: OSA

Muhammad Rodlin Billah and co-workers from Germany have presented an efficient way to connect III-V light sources to passive silicon photonic circuits. Their approach, photonic wire-bonding, essentially relies on direct-write twophoton lithography to fabricate in situ three-dimensional freeform waveguides between optical chips for hybrid integration. Highly efficient coupling between indium phosphide -based horizontal-cavity surface-emitting lasers and photonic chips, through a three-dimensionally shaped photonic wire bond with a size of $2.0 \mu \mathrm{m} \times 1.4 \mu \mathrm{m}$, was achieved with coupling losses down to $0.4 \mathrm{~dB}$. The bonds were fabricated from a commercially available negative-tone photoresist and can be adapted to the exact positions of the chips such that high-precision alignment of chips is not imperative, hence allowing automated processes for mass production.

https://doi.org/10.1038/s41566-018-0251-Z

\section{FUNDAMENTAL OPTICS}

\section{Slow and steady}

Nat. Phys. http://doi.org/csw3 (2018)

An understanding of the maximum possible enhancement of spontaneous electron radiation is useful for many types of light-emitting device. Yi Yang and co-workers from the USA and Israel have now theoretically derived and experimentally confirmed the upper limit for spontaneous photon emission and energy loss of free electrons, regardless of geometry. The limit is bound mainly by intrinsic loss, electron velocity and an 'impact parameter', but no other structural details. The impact parameter is a scale parameter that is also proportional to Lorentz contraction. While it is expected that electrons in the far field of a structure (and impact parameter is much greater than unity) yield stronger photon emission, surprisingly, if electrons are in the near field (and impact parameter is much smaller than unity), slower electrons are optimal. Physically, this possibility is actually intuitive; slower electrons have more opportunity to build up stronger near-fields on the structures.

DFPP
THERMOPHOTONICS Waste-heat recovery

Thermal electromagnetic radiation can be converted with relatively high efficiency thanks to photovoltaic technology, but the power density of thermal radiation is low compared with what is possible with the conduction-based generators. Bo Zhao and colleagues from the USA tackle this problem by proposing a device that exploits evanescent fields that may offer simultaneously high power density $\left(9.6 \mathrm{~W} \mathrm{~cm}^{-2}\right)$ and thermal-to-electrical conversion efficiency (9.8\%). Their solid-state thermophotonic system uses an AIGaAs light-emitting diode (LED) (hot side) and an AIGaAs photovoltaic (PV) cell (cold side), both $900 \mathrm{~nm}$ thick, with $\mathrm{Ag}$ on the back-sides of each acting as reflectors, critical for the achieved efficiency. Emission from the LED is captured by the PV cell and some of the generated power biases and drives the LED. The trick to achieving high power density is working in a near-field regime with a gap spacing of only $10 \mathrm{~nm}$ between the $600 \mathrm{~K}$ hot side and the $300 \mathrm{~K}$ cold side, as well as appropriate layer thicknesses to reduce non-radiative recombination. This temperature range represents what is known as 'low-grade' heat waste where thermoelectric devices dominate, but the proposed thermophotonic device may outperform these.

\section{SINGLE-PHOTON DEVICES}

\section{Solid-state success \\ Science 361, 57-60 (2018)}

RW Shuo Sun and co-workers from the USA have realized a single-photon switch and transistor using a solid-state quantum memory. The device consists of a semiconductor spin qubit strongly coupled to a nanophotonic cavity that is formed by a two-dimensional GaAs photonic crystal (PC), with a three-hole defect, containing a single layer of InAs quantum dots. A magnetic field of $5.5 \mathrm{~T}$ is applied to the $\mathrm{PC}$ so that one of the four optical transitions is resonant with the PC defect-cavity; a gate pulse and a signal optical pulse are resonant with the PC defect-cavity. The gate pulse first sets the spin state as a quantum memory of the switch, and the spin state is subsequently read by the transmittance contrast of the signal pulse. The spin qubit consists of a single electron in a charged InAs quantum dot. The team reports that the spin qubit enables a single 63-ps gate photon to switch a signal field containing up to an average of 27.7 photons. The maximum gain of 3.3 is obtained for a signal field containing an average of 29.2 photons.

https://doi.org/10.1038/s41566-018-0249-6

\section{LIQUID CRYSTALS}

\section{Tunable circuits}

\section{ACS Photon. 5, 3222-3230 (2018)}

By exploiting the capability of direct laser writing on different photopolymerizable materials on the same chip and the versatile properties of different polymers, Sara Nocentini and colleagues from Italy have demonstrated a three-dimensional active and tunable polymeric integrated network for telecommunications at $1,550 \mathrm{~nm}$. They designed, fabricated and characterized straight and bent waveguides, grating couplers, and single and vertically coupled whispering-gallery-mode (WGM) resonators. Passive, rigid photonic components were first fabricated on a low-refractive-index fused silica substrate. To realize tunable photonic resonators, the team integrated liquid-crystalline networks (LCNs) into the photonic circuit. The shape change and the refractive-index variation due to the optically induced rearrangement of LCN polymeric chains were exploited in two different ways to tune the photonic resonators - LCNs were used as an actuator to finely change the shape of the WGM resonators and were also used to make the resonator itself.

$R W$ 